\title{
A Laser Fabrication of Magnetic Micromachines by Using Optimized Photosensitive Ferrofluids
}

\author{
Ye Tian and Xiangxin Shao \\ College of Electrical and Electronic Engineering, Changchun University of Technology, Changchun 130012, China \\ Correspondence should be addressed to Xiangxin Shao; shaoxiangxin@163.com
}

Received 22 March 2016; Accepted 26 May 2016

Academic Editor: Paulo Cesar Morais

Copyright (C) 2016 Y. Tian and X. Shao. This is an open access article distributed under the Creative Commons Attribution License, which permits unrestricted use, distribution, and reproduction in any medium, provided the original work is properly cited.

\begin{abstract}
We report here a laser fabrication of magnetic micromachines by using optimized photosensitive ferrofluids. $\mathrm{Fe}_{3} \mathrm{O}_{4}$ nanoparticles were prepared by thermal decomposition and subsequent ligand exchange. And then, they were dispersed into photoresist. As a representative illustration, a magnetic microturbine with high surface flatness was fabricated, and its rotation speed could reach as high as $400 \mathrm{rpm}$ under revolving magnetic field.
\end{abstract}

\section{Introduction}

Laser micronanofabrication technology through two-photon photopolymerization (TPP) of resins has emerged as an interesting tool for the fabrication of micromachines due to their reasonably high spatial resolution and three-dimensional (3D) processing capability [1-5]. To date, various micronanodevices such as moveable needle pairs [6], microoscillators [7], microspring [8], and microrotators [9] have been successfully fabricated through the laser processing. However, it is very difficult to manipulate these tiny devices in a controlled manner. At present, a vast majority of them are manipulated by optical driving manner which needs a sharply focused laser $[6,10]$. The half-contact of this mechanism limits the applications of micromachines in some special fields, for example, operating a micromachine in blood vessel for thrombolytic therapy. Therefore, an appropriate driving mechanism is highly desired [11-14]. Here we solved the problem by doping surface modified $\mathrm{Fe}_{3} \mathrm{O}_{4}$ nanoparticles in photopolymerizable resins, from which magnetic micromachines are produced by TPP. Then, remote control of these micromachines is readily realized by external magnetic field. Finally, as an example of a promising application, a microturbine was fabricated in the intersection of a "Y-" shape microfluidic channel. It would work as an active mixer in magnetic field.

\section{Experimental}

Monodisperse $\mathrm{Fe}_{3} \mathrm{O}_{4}$ nanoparticles were synthesized using thermal decomposition method [15]. $2 \mathrm{mmol} \mathrm{Fe}(\mathrm{acac})_{3}$, $10 \mathrm{mmol}$ 1,2-hexadecanediol, $6 \mathrm{mmol}$ oleic acid, $6 \mathrm{mmol}$ oleylamine, and $20 \mathrm{~mL}$ phenyl ether were mixed and magnetically stirred under a blanket of nitrogen. The mixture was heated to $200^{\circ} \mathrm{C}$ and stayed for $30 \mathrm{~min}$ and heated to reflux $\left(300^{\circ} \mathrm{C}\right)$ for $2 \mathrm{~h}$. And then it was cooled to room temperature. When $40 \mathrm{~mL}$ ethanol was added to the mixture, a black material was precipitated and separated by centrifugation $(7000 \mathrm{rpm}, 10 \mathrm{~min})$. The black product was then dissolved in $20 \mathrm{~mL}$ hexane. Centrifugation $(9000 \mathrm{rpm}$, $10 \mathrm{~min}$ ) was applied to remove any undispersed residue. The product, $6 \mathrm{~nm} \mathrm{Fe}_{3} \mathrm{O}_{4}$ nanoparticles, was then precipitated with ethanol, centrifuged (7000 rpm, $10 \mathrm{~min}$ ) to remove the solvent, and redispersed into butyl methacrylate.

For improving their compatibility in photoresists, surface modification process was performed. Typically, $100 \mathrm{mg}$ of oleic acid stabilized $\mathrm{Fe}_{3} \mathrm{O}_{4}$ nanoparticles was dispersed in $20 \mathrm{~mL}$ of propoxylated trimethylolpropane triacrylate $\left(\mathrm{PO}_{3}-\right.$ TMPTA, a kind of cross-linker) and stirred for $24 \mathrm{~h}$ at room temperature. After that the nanoparticles were dispersed homogeneously in $\mathrm{PO}_{3}$-TMPTA. Subsequently, the nanoparticles were washed with ethanol for three times with the help of a magnet. The nanoparticles were kept 


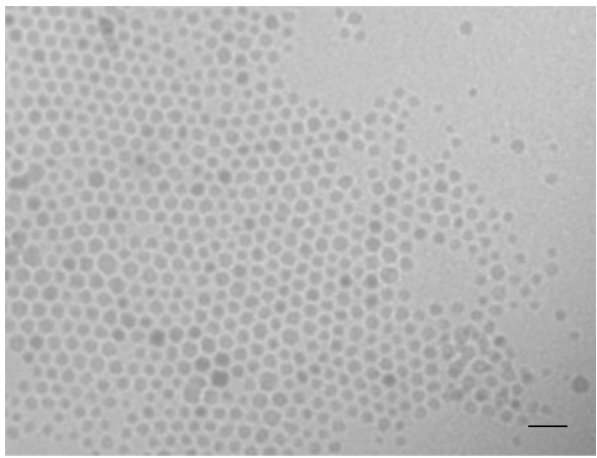

(a)

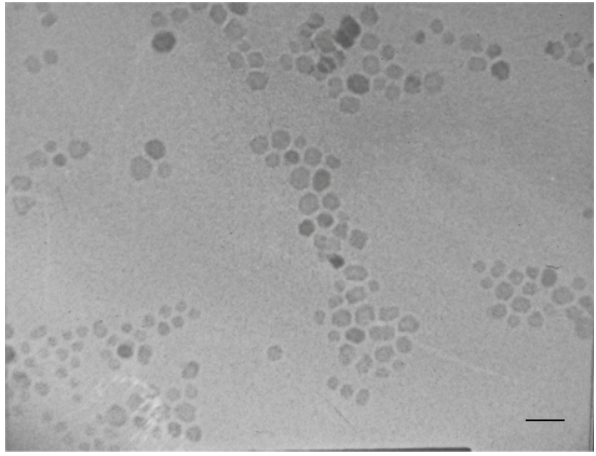

(b)

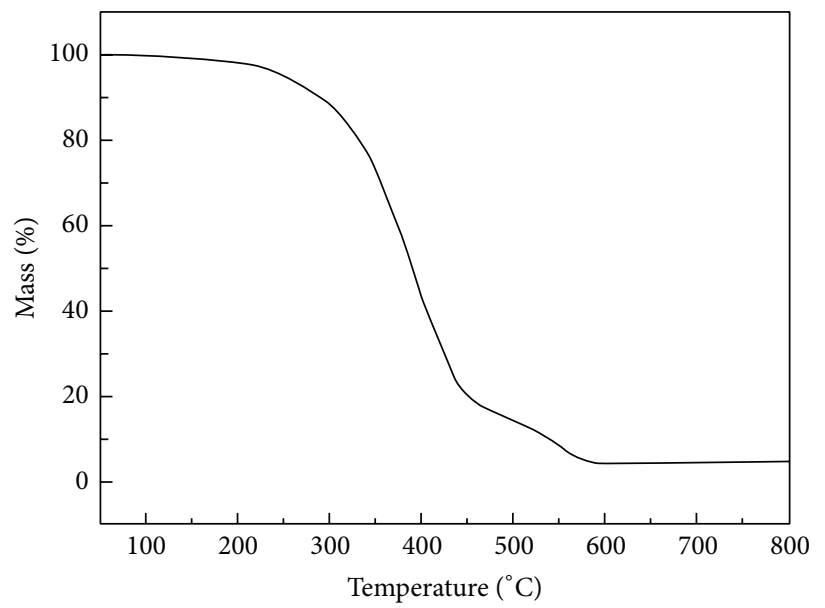

(c)

FIgURE 1: TEM images of different $\mathrm{Fe}_{3} \mathrm{O}_{4}$ nanoparticles. (a) Oleic acid stabilized $\mathrm{Fe}_{3} \mathrm{O}_{4}$ nanoparticles prepared using thermal decomposition method. (b) $\mathrm{PO}_{3}$-TMPTA modified $\mathrm{Fe}_{3} \mathrm{O}_{4}$ nanoparticles. The scales in images are $20 \mathrm{~nm}$. (c) TGA curve of the photosensitive ferrofluid.

in butyl methacrylate under the protection of $\mathrm{N}_{2}$. After ligand exchange process, $\mathrm{PO}_{3}$-TMPTA was modified on the surface of nanoparticles, and then the nanoparticles could be homogeneously dispersed into acrylate-based photopolymerizable resin consisting of butyl methacrylate (36 wt.\%) as monomer, $\mathrm{PO}_{3}$-TMPTA (56 wt.\%) as cross-linker, and 2,4,6-trimethylbenzoyldiphenylphosphinoxid (4wt.\%) and phenylbis (2,4,6-trimethylbenzoyl) phosphine oxide (4 wt.\%) as photoinitiators.

Using this ferrofluid photoresist, microturbines were fabricated by pinpoint laser writing. In this work, femtosecond laser pulses were tightly focused into the resin by a $60 \mathrm{x}$ oil immersion objective lens with a high numerical aperture (NA $=1.35$ ). The central wavelength of the laser was $790 \mathrm{~nm}$ with a pulse width of $120 \mathrm{fs}$ and a repetition rate of $80 \mathrm{MHz}$. The laser focal spot point was scanned point by point in the lateral dimensions by a galvano mirror pair and in the lengthwise dimension by a piezo stage. The scanning step length was $100 \mathrm{~nm}$. The polymerization was induced under the condition of $6.5 \mathrm{~mW}$ laser power before the objective lens and of $500 \mathrm{~ms}$ exposure duration at each dot. The complete process of fabrication was controlled exactly by computer. Finally, the whole structures were immersed in ethanol for $1 \mathrm{~min}$ to remove the unpolymerized resin, leaving a solidified skeleton.

\section{Results and Discussion}

In order to evaluate the particle size and uniformity, both as-synthesized and modified $\mathrm{Fe}_{3} \mathrm{O}_{4}$ nanoparticles are characterized by TEM (Figures 1(a) and 1(b)). Statistical results show that nanoparticles before and after modification are well monodispersed and homogeneous. The average diameter of as-synthesized nanoparticles is about $6 \mathrm{~nm}$. After surface modification, a slight increase to $\sim 8 \mathrm{~nm}$ is observed. Thermogravimetric analysis (TGA) measurement gives the direct evidence of surface modification. Figure 1(c) is TGA curve of the photosensitive ferrofluid. There is a two-step mass loss in the TGA curve. The first mass loss of $82 \mathrm{wt} . \%$ from $200^{\circ} \mathrm{C}$ to $450^{\circ} \mathrm{C}$ is due to the thermal decomposition of photoresist, and the second mass loss of $13.5 \mathrm{wt} . \%$ from $450^{\circ} \mathrm{C}$ to $570^{\circ} \mathrm{C}$ is attributed to the thermal decomposition of chemisorbed 6-(methacryloyloxy) hexanoic acid on particles surfaces. The remaining mass of $4.5 \mathrm{wt} . \%$ is attributed to $\mathrm{Fe}_{3} \mathrm{O}_{4}$ in the resin.

The concentration of $\mathrm{Fe}_{3} \mathrm{O}_{4}$ nanoparticles in the resin mixture is crucial. The turbine could not correspond to the rotating external magnetic field when the concentration is too low. However, when the nanoparticles concentration is too high, the scattering and linear absorption of nanoparticles will highly increase, which would lead to more roughness of 


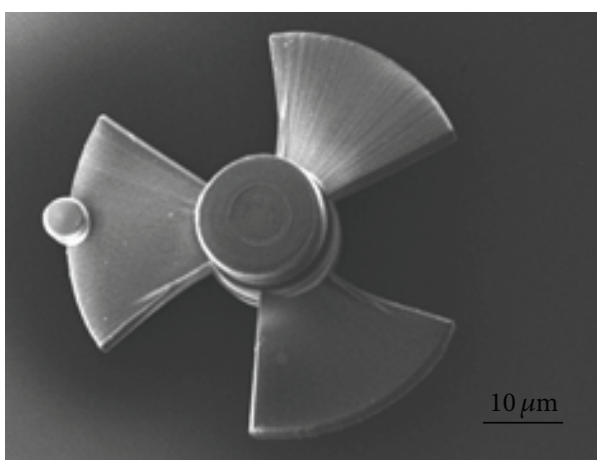

(a)

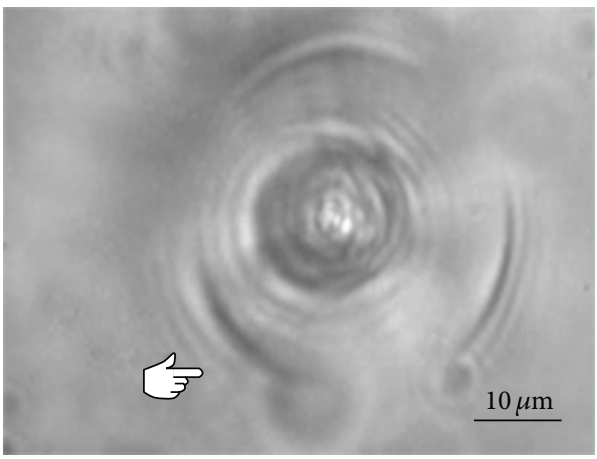

(c)

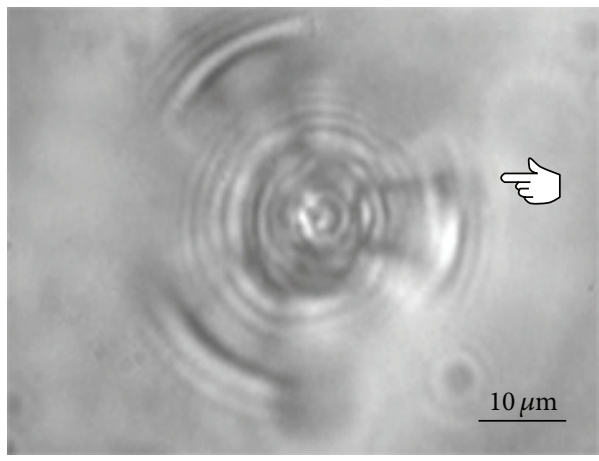

(e)

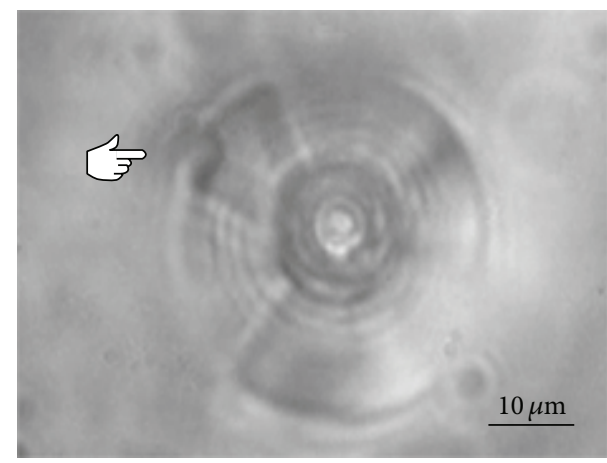

(b)

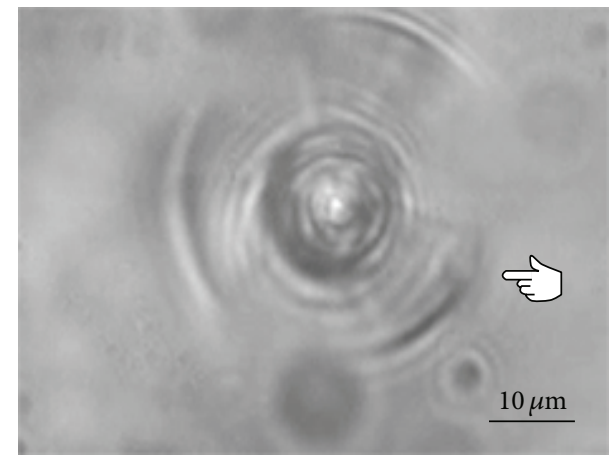

(d)

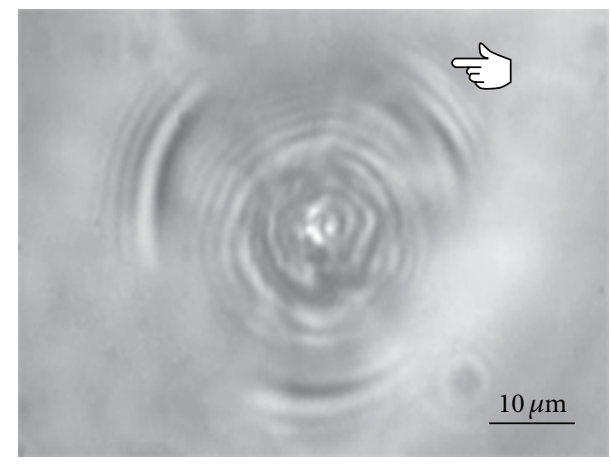

(f)

FIGURE 2: Remote control of microturbine. (a) SEM image of $\mathrm{Fe}_{3} \mathrm{O}_{4}$ nanoparticle-contained microturbine. (b)-(f) Optical microscopy images of the microturbine in a circumgyratetion cycle. The positions of the point on the turbine blade were marked in the images.

the microdevices. In the experiments, the concentration of $\mathrm{Fe}_{3} \mathrm{O}_{4}$ in the resin mixture is kept from 4 wt.\% to 5 wt.\%.

As a representative illustration, microturbines are created by TPP of the ferrofluid photoresist (Figure 2(a)). Because the modification of nanoparticles enhances the compatibility between nanoparticles and photoresist, the surface of microturbines is much smoother. As a result, when the microturbines rotate, the surface friction of them decreases significantly, and the stability and efficiency of microturbines are improved evidently. Figures 2(b)-2(f) are optical microscopy images of the microturbine in a circumgyratetion cycle. For remote control of the microturbine, a piece of ferromagnet is placed on a vortical device around the objective lens which could provide about 4000 gauss magnetic field. For observation of the microturbine working, a point was fabricated on the turbine blade. Due to the fast speed of rotation, the images of the point become a little fuzzy. The maximum rotating speed of the microturbines containing modified $\mathrm{Fe}_{3} \mathrm{O}_{4}$ nanoparticles is as high as $400 \mathrm{r} / \mathrm{min}$.

The fabrication of such magnetic microdevices is not limited to flat substrates. As shown in Figure 3, a magnetic microturbine is successfully prepared in the intersection of a "Y-" shape microfluidic channel. It is well known that mixing is very difficult in microscale. Such a complex threedimensional structure could be regarded as a passive mixer in the channel. When it rotates in magnetic field, the turbine could be regarded as an active mixer. As an effectively mixing device, it could be used to solve the problem of solvents mixing in microscale. 


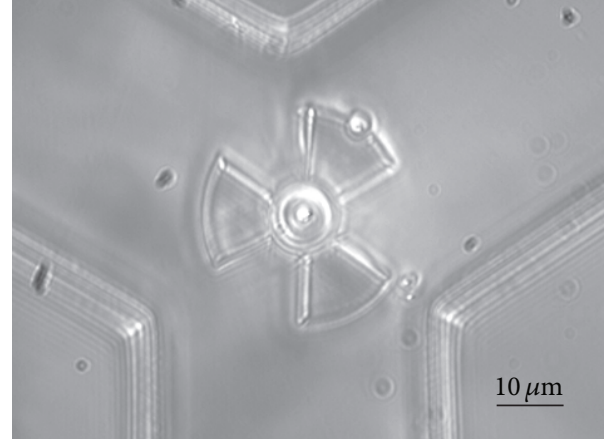

FIgURE 3: Microturbine fabricated with magnetic photoresist containing $\mathrm{PO}_{3}$-TMPTA modified $\mathrm{Fe}_{3} \mathrm{O}_{4}$ nanoparticles in the "Y-" shape channel of a microchip.

\section{Conclusions}

In summary, we have successfully prepared a photosensitive ferrofluid by homogeneously doping the surface modified $\mathrm{Fe}_{3} \mathrm{O}_{4}$ nanoparticles into acrylate-based photopolymers. The ferrofluid photoresist was utilized to fabricate high quality microturbines for remote manipulation under external magnetic field.

\section{Competing Interests}

The authors declare that they have no competing interests.

\section{References}

[1] J.-F. Xing, M.-L. Zheng, and X.-M. Duan, “Two-photon polymerization microfabrication of hydrogels: an advanced 3D printing technology for tissue engineering and drug delivery," Chemical Society Reviews, vol. 44, no. 15, pp. 5031-5039, 2015.

[2] C.-L. Lin, Y.-J. Liu, Z.-D. Lin et al., "Laser direct writing 3D structures for microfluidic channels: flow meter and mixer," in Microfluidics, BioMEMS, and Medical Microsystems XIII, vol. 9320 of Proceedings of SPIE, San Francisco, Calif, USA, February 2015.

[3] V. Tribuzi, R. D. Fonseca, D. S. Correa, and C. R. Mendonça, "Birefringent microstructures fabricated by two-photon polymerization containing an azopolymer," Optical Materials Express, vol. 3, no. 1, pp. 21-26, 2013.

[4] K. Masui, S. Shoji, K. Asaba et al., "Laser fabrication of Au nanorod aggregates microstructures assisted by two-photon polymerization," Optics Express, vol. 19, no. 23, pp. 2278622796, 2011.

[5] Y.-L. Zhang, Q.-D. Chen, H. Xia, and H.-B. Sun, "Designable 3D nanofabrication by femtosecond laser direct writing," Nano Today, vol. 5, no. 5, pp. 435-448, 2010.

[6] S. Maruo, K. Ikuta, and H. Korogi, "Submicron manipulation tools driven by light in a liquid," Applied Physics Letters, vol. 82, no. 1, pp. 133-135, 2003.

[7] H.-B. Sun, K. Takada, and S. Kawata, "Elastic force analysis of functional polymer submicron oscillators," Applied Physics Letters, vol. 79, no. 19, pp. 3173-3175, 2001.

[8] Y. Tian, D. Lu, H. Jiang, and X. Lin, "Preparation of a novel ferrofluidic photoresist for two-photon photopolymerization technique," Journal of Magnetism and Magnetic Materials, vol. 324, no. 20, pp. 3291-3294, 2012.

[9] P. Galajda and P. Ormos, "Rotors produced and driven in laser tweezers with reversed direction of rotation," Applied Physics Letters, vol. 80, no. 24, pp. 4653-4655, 2002.

[10] Y. W. Zong, J. Liu, R. Liu et al., "An optically driven bistable janus rotor with patterned metal coatings," ACS Nano, vol. 9, no. 11, pp. 10844-10851, 2015.

[11] Y.-J. Yum, H. Hwang, M. Kelemen, V. Maxim, and P. Frankovský, "In-pipe micromachine locomotion via the inertial stepping principle," Journal of Mechanical Science and Technology, vol. 28, no. 8, pp. 3237-3247, 2014.

[12] Y. Tian, Y.-L. Zhang, H. Xia et al., "Solvent response of polymers for micromachine manipulation," Physical Chemistry Chemical Physics, vol. 13, no. 11, pp. 4835-4838, 2011.

[13] S. Campuzano, D. Kagan, J. Orozco, and J. Wang, "Motiondriven sensing and biosensing using electrochemically propelled nanomotors," Analyst, vol. 136, no. 22, pp. 4621-4630, 2011.

[14] A. J. Nichol, P. S. Stellman, W. J. Arora, and G. Barbastathis, "Two-step magnetic self-alignment of folded membranes for 3D nanomanufacturing," Microelectronic Engineering, vol. 84, no. 5-8, pp. 1168-1171, 2007.

[15] S. H. Sun and H. Zeng, "Size-controlled synthesis of magnetite nanoparticles," Journal of the American Chemical Society, vol. 124, no. 28, pp. 8204-8205, 2002. 

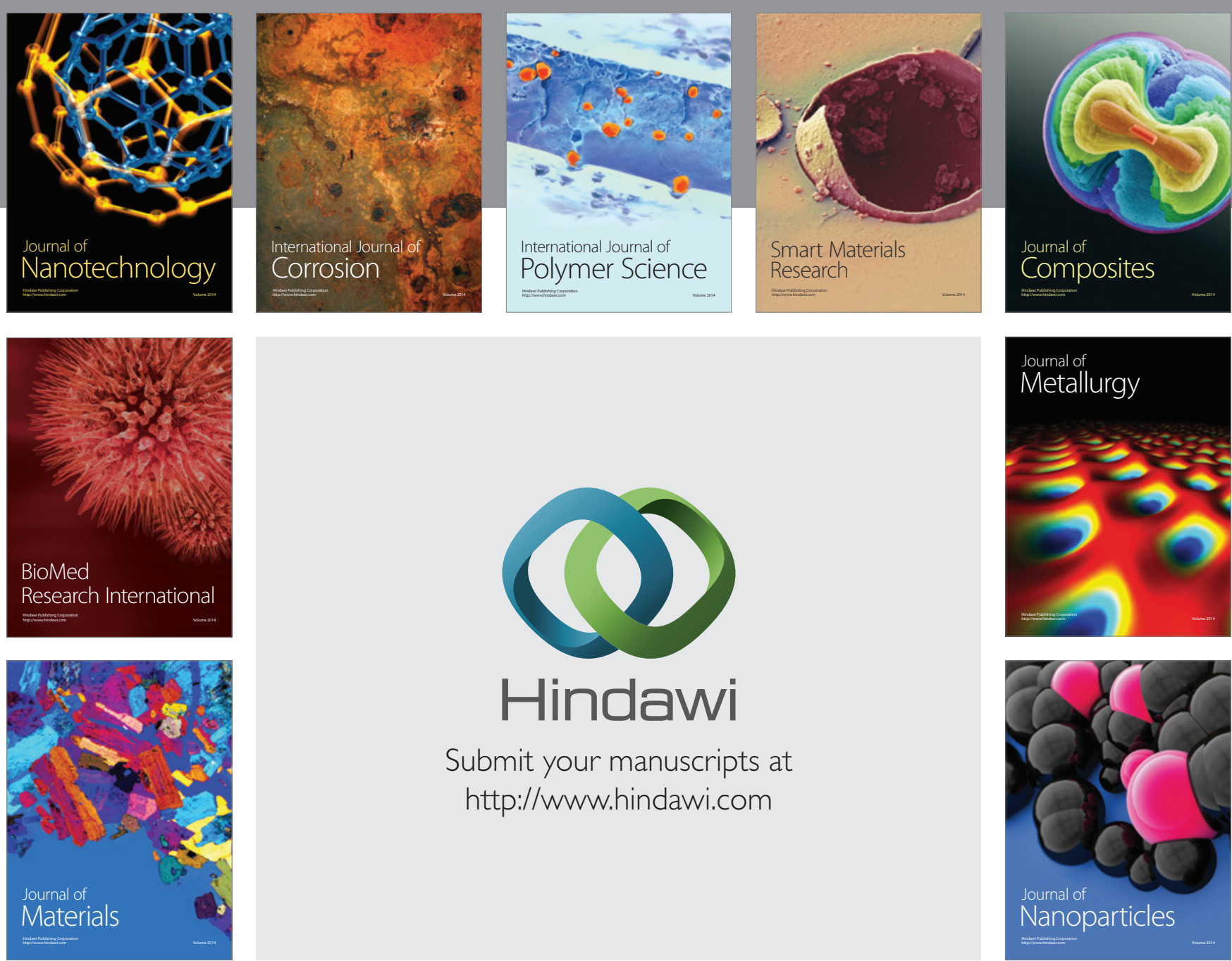

\section{Hindawi}

Submit your manuscripts at

http://www.hindawi.com

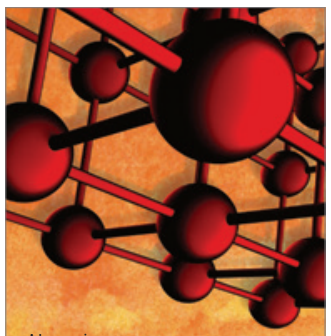

Materials Science and Engineering
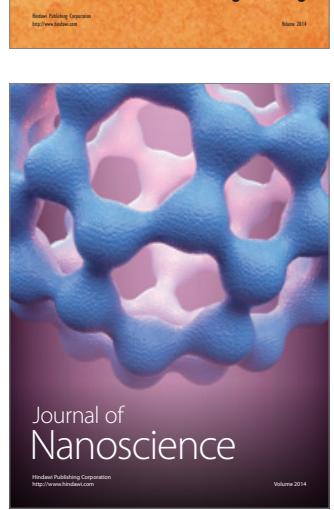
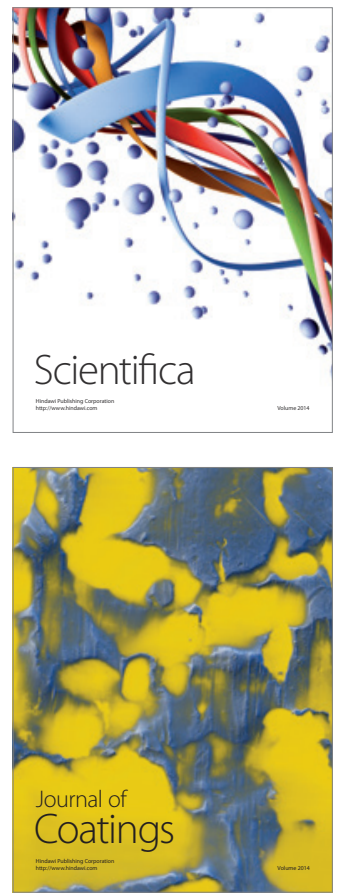
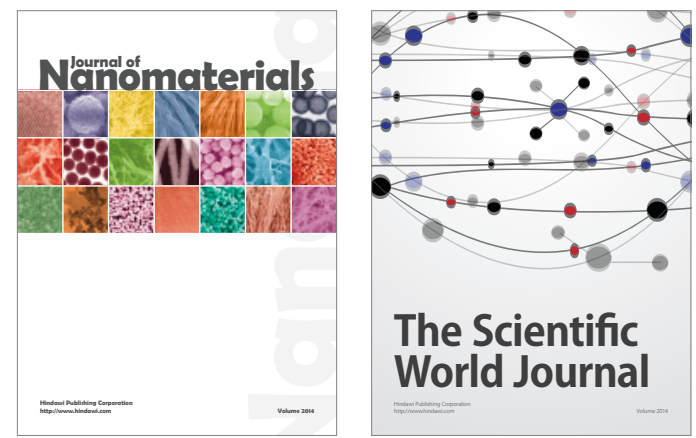

The Scientific World Journal
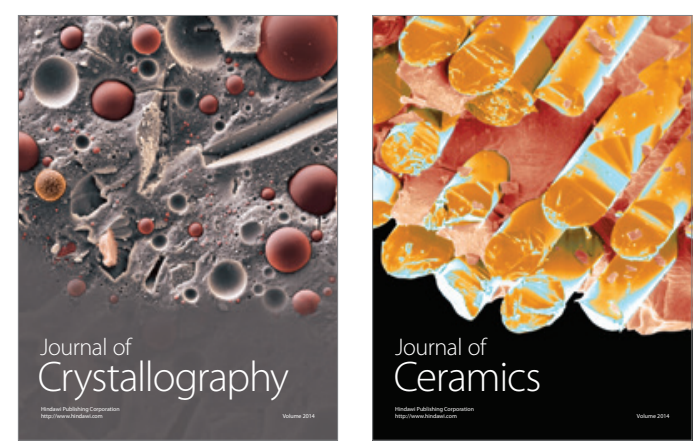
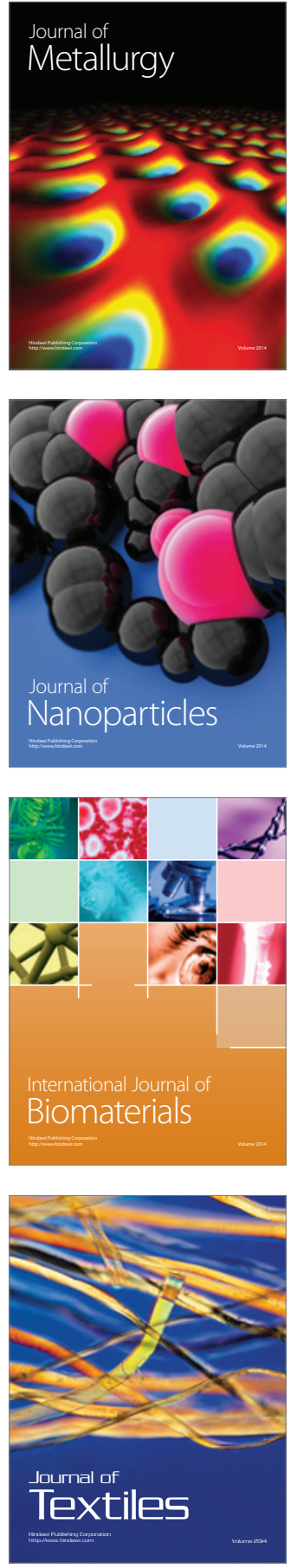